\title{
Self-consistent three-dimensional computations of non-axisymmetric ITER equilibria
}

\author{
E. Strumberger, S. Günter, P. Merkel, E. Schwarz, and C. Tichmann \\ Max-Planck-Institut für Plasmaphysik, IPP-Euratom Association \\ 85748 Garching, Germany
}

\begin{abstract}
Three planned Test Blanket Modules (TBMs) and 18 toroidal field coils break the axisymmetry of the ITER magnetic field. In this paper, the plasma response on these non-axisymmetric fields is studied quantitatively. For this purpose, self-consistent, three-dimensional, free-boundary equilibria of type ITER scenario 4 are computed. The resulting 3D equilibrium magnetic fields are then compared with the corresponding axisymmetric fields to which the vacuum perturbation fields are superimposed. The studies are performed for various normalized plasma pressures.
\end{abstract}

PACS numbers: $52.20 \mathrm{Dq}, 52.35 \mathrm{Py}, 52.55 \mathrm{Dy}, 52.55 \mathrm{Fa}, 52.55 \mathrm{Tn}, 52.65 \mathrm{Kj}$ 


\section{Introduction}

Tokamaks are usually considered to be axisymmetric. However, the finite number of toroidal field coils results in a toroidal field ripple that breaks the axisymmetry. Besides the field ripple, the symmetry may be broken because of a number of effects, such as (i) error fields produced by misaligned coils, e.g. [1, 2], (ii) additional resonant magnetic perturbation fields, e.g. ELM (Edge Localized Mode) control fields [3, 4], (iii) installed ferritic inserts which reduce the field ripple but break the toroidal symmetry [5, 6], (iv) asymmetrically placed Test Blanket Modules (TBM) used to test tritium breeding [7], and/or (v) wall structures with holes and port extensions $[8,9]$ close to the plasma.

While collisionless, trapped particles are confined in axisymmetric fields, field perturbations lead to a loss of these particles due to stochastic diffusion and/or ripple well trapping [10, 11]. Fast particle losses (3.5 MeV $\alpha$-particles and $1 \mathrm{MeV}$ neutral beam particles) in ITER caused by toroidal field ripple and perturbation fields are currently subject of several investigations $[7,12,13]$. The 3D magnetic fields used in these studies are superpositions of axisymmetric equilibrium fields and 3D perturbation fields. The latter are the vacuum ripple field created by the 18 toroidal field coils, and the fields produced by TBMs and ferritic inserts (FI) [7]. In the current conceptual design, the ferritic inserts are placed in the gap between the inner and outer wall of the ITER vacuum vessel in each of the 18 toroidal sections. The three TBMs shall be installed in the equatorial ports \#2, 16 and 18. That is, they will be located in one quadrant of the torus at toroidal angles $\phi=30,310$ and $350^{\circ}$, respectively. A more detailed description of TBMs and FIs, and the computation of their magnetic field contributions can be found in [7]. Note that in these calculations the fields produced by the axisymmetric plasma currents have also been included.

Superimposing vacuum perturbation fields to the axisymmetric equilibrium field is a standard numerical procedure for tokamaks. However, for many years it is an subject of several investigations $[14,15,16,17]$ whether the plasma currents are significantly changed by these perturbation fields. In order to address the significance of these changes for ITER equilibrium configurations, we compute 3D free-boundary equilibria of type scenario 4, taking into account the uncompensated field ripple, and the influence of FIs and TBMs. The resulting 3D equilibrium fields, that is, their geometry and ripple, are then compared with the corresponding axisymmetric equilibrium fields to which the perturbation fields have been added. We investigate equilibria with various volume-averaged $\beta$-values $(<\beta>=2.24,3.66$, and $4.67 \%)$, because the plasma response is expected to be $\beta$-dependent. The volume-averaged plasma beta is defined by $\left\langle\beta>=2 \mu_{0}<p>/<B^{2}>\right.$ with $p$ and $B$ being plasma pressure and magnetic field 
strength, and $<>$ indicating the volume average. The numerical calculations of the freeboundary equilibria are performed with the VMEC/NEMEC code [18, 19]. Assuming nested flux surfaces the code minimizes the total energy of a plasma confined in a toroidal domain. The vacuum magnetic field, the total toroidal flux, and the pressure and safety factor serve as input to the code.

The paper is organized as follows. In Sect. 2, the ITER equilibrium scenario 4 and the design of similar equilibria with higher plasma beta values are described. Then, in Sect. 3, the toroidal field ripple is investigated for the considered $\beta$-values, and in Sect. 4, the effects of TBMs and FIs are studied. Finally, a summary and conclusions are presented in Sect. 5.

\section{ITER equilibria}

Our studies are based on the steady state ITER scenario 4 with a toroidal plasma current of 9 MA, a volume-averaged plasma beta of $\langle\beta\rangle=2.24 \%$, a weak negative magnetic shear and a strongly shaped plasma. This configuration is assumed to produce $300 \mathrm{MW}$ of fusion power with $Q=5$ for $3000 \mathrm{~s}$. The plasma shape of the axisymmetric equilibrium, as well as the profiles of pressure, $p$, and safety factor, $q$, are shown in Fig. 1 (solid curves).

In order to study the influence of the plasma beta on the plasma response, we designed in addition two similar equilibria with $\langle\beta\rangle=3.66$ and $4.67 \%$. These equilibria have identical $q$-profiles, self-similar pressure profiles and almost identical plasma boundaries, as illustrated in Fig. 1. Keeping the total toroidal flux and the $q$-profile fixed leads to slightly increased toroidal plasma currents $I_{\mathrm{p}}=-9.50 \mathrm{MA}(<\beta>=3.66 \%)$ and $I_{\mathrm{p}}=-9.87 \mathrm{MA}(<\beta>=4.67 \%)$. Increasing the plasma pressure also rises the Shafranov shift. The magnetic axis is shifted from $R_{\mathrm{a}}=6.703 \mathrm{~m}, Z_{\mathrm{a}}=0.515 \mathrm{~m}$ to $R_{\mathrm{a}}=6.829 \mathrm{~m}, Z_{\mathrm{a}}=0.506 \mathrm{~m}$ and $R_{\mathrm{a}}=6.900 \mathrm{~m}, Z_{\mathrm{a}}=0.504 \mathrm{~m}$, respectively.

In order to fix the plasma boundary, it is necessary to adjust the currents in the poloidal field coils which are responsible for plasma shape and position. In a first step, axisymmetric fixedboundary equilibria with the selected plasma beta values are computed. Then, these equilibria are used to determine the required poloidal coil currents with the CURFIT (CURrent FIT) code, which has been developed from the NESCOIL code [20]. The CURFIT code determines the poloidal coil currents which produce a poloidal magnetic field surrounding the plasma equilibrium and satisfying the boundary conditions at the plasma boundary. The poloidal coil currents of the standard ITER scenario 4 and the two higher $\beta$ equilibria are listed in Table 1. A comparison shows that small current changes in some of the poloidal field coils are sufficient to retain position and shape of the plasma boundary. 


\section{Toroidal field ripple}

The 18 toroidal field coils of ITER produce a maximum toroidal field ripple of approximately $1.1 \%$ at the plasma boundary on the low-field side. In this study, we compute the ripple with two different methods. In the first one, the standard tokamak method is used. That is, axisymmetric equilibria and their corresponding fields are computed on which the vacuum ripple field is superimposed. The latter is defined as $3 \mathrm{D}$ vacuum field minus axisymmetric vacuum field. The second method corresponds to the computation of 3D free-boundary equilibria where the ripple is taken into account self-consistently. The ripple of the resulting $3 \mathrm{D}$ equilibrium field is compared with the one obtained with the first method for all $\beta$-values considered. In the following, we will denote the ripples computed with the first and the second method with $\delta_{\mathrm{v}}$ and $\delta_{\mathrm{e}}$, respectively. Both are defined by

$$
\delta=\frac{B_{\max }-B_{\min }}{B_{\max }+B_{\min }}
$$

with $B_{\max }$ and $B_{\min }$ being the maximum and minimum field strength values in toroidal direction. Figure $2 \mathrm{a}$ shows $\delta_{\mathrm{v}}$ for $\langle\beta\rangle=2.24 \%$, while Fig. $2 \mathrm{~b}$ presents the difference $\Delta \delta=\delta_{\mathrm{e}}-\delta_{\mathrm{v}}$ of the field ripple obtained by the two methods. Furthermore, the ripple differences for $\langle\beta\rangle=3.66$ and $4.67 \%$ are presented in Figs $2 \mathrm{c}$ and $2 \mathrm{~d}$. The figures clearly show that the differences $\Delta \delta$ increase with increasing $\beta$. However, even for $\langle\beta\rangle=4.67 \%$ the differences between the two methods (the plasma currents increase the ripple) remain negligible. The maximum differences between $\delta_{\mathrm{e}}$ and $\delta_{\mathrm{v}}$ amount to $3.9 \%$ of the ripple value $\delta_{\mathrm{v}}$ for $\langle\beta\rangle=2.24 \%, 6.3 \%$ for $\langle\beta\rangle=$ $3.66 \%$, and $8.2 \%$ for $\langle\beta\rangle=4.67 \%$. These differences are measured in the outer midplane at $Z=0.5 \mathrm{~m}$ and $R=7.8-7.9 \mathrm{~m}$.

The $\beta$-dependent modifications of the ripple are caused by the toroidal and poloidal plasma currents. As discussed in detail in papers by J.L. Johnson et al. [14] and Y. Suzuki et al. [15], the field ripple is modified by the ripple of these currents. At the positions of the toroidal field coils the ripple of the toroidal current density is maximum whereas the ripple of the poloidal current density is minimum. Furthermore, the plasma currents (Pfirsch-Schlüter and diamagnetic currents) are proportional to the pressure gradient. Therefore, the patterns of ripple difference $\Delta \delta$, which arise in Figs 2b, c and d, are related to the pressure gradient as is demonstrated in Fig. 3 . There, an enlargement of the upper part of the outer midplane of Fig. $2 d(<\beta>=4.67 \%)$ is shown. Additionally, the figure includes the pressure profile as function of the radial co-ordinate (solid curve). As expected, the plasma response to the field ripple is maximum in regions of high ripple field and steep pressure gradients.

The ripple causes small toroidal variations of the flux surfaces: at $R=7.88 \mathrm{~m}, Z=0.50 \mathrm{~m}$ it is $\approx 2.1 \mathrm{~mm}$ and increases to $\approx 5 \mathrm{~mm}$ at the plasma boundary. These variations are negligible in 
comparison with a major radius of $R_{0}=6.35 \mathrm{~m}$ and a minor radius of $a=1.86 \mathrm{~m}$. In Fig. $4 \mathrm{a}$ the variation of a flux surface is illustrated for $\langle\beta\rangle=4.67 \%$. This plot is an enlargement of the outer midplane region showing the position of the flux surface at $\varphi=0$ (left), $\varphi=5$ (middle/red), $\varphi=10$ (right), and $\varphi=15^{\circ}$ (middle/blue) resulting from the 3D free-boundary equilibrium computation. In Fig. $4 \mathrm{~b}$ this flux surface is compared with a Poincaré plot representing a field line plotted at the same cross-sections, but traced in the corresponding axisymmetric equilibrium field with superimposed vacuum ripple field. The field line shows the same radial shift as the flux surface of the 3D equilibrium. Here it should be noted that the toroidal ripple only bends flux surfaces, but it doesn't create islands at flux surfaces with rational $q$-values. This is a consequence of the high toroidal mode number of $n \geq 18$ of the ripple perturbation field. The situation is different in case of the three TBMs, as we will show in the next section.

\section{Ferritic inserts and TBMs}

Ferritic inserts and TBMs alter the magnetic field. The corresponding field perturbation has been computed by K. Shinohara et al. [7]. In Fig. 5, we show the components $\left(b_{\varphi}, b_{\mathrm{R}}, b_{\mathrm{Z}}\right)$ of the vacuum ripple field (solid curve) and the ripple field modified by FIs and TBMs (dashed curve) as function of the toroidal angle $\varphi$ for a representative co-ordinate point $(R=8.2 \mathrm{~m}$, $Z=0.5 \mathrm{~m}$ ). The toroidal field coils and ferritic inserts produce a magnetic field with 18 periods, but the TBMs break this symmetry. In Fig. 5, the three spikes around $\varphi=30,310$ and $350^{\circ}$ are generated by the TBMs, which are placed in these toroidal regions.

Using plasma boundary and flux co-ordinates $(s, u, v)$ given by the VMEC/NEMEC code, a Fourier decomposition of the modified ripple field on this flux surface $(s=1)$ is made

$$
b_{\mathrm{i}}=\sum_{\mathrm{m}, \mathrm{n}} b_{\mathrm{i}, \mathrm{m}, \mathrm{n}}^{\mathrm{c}} \cos (2 \pi(\mathrm{m} u+\mathrm{n} v))+b_{\mathrm{i}, \mathrm{m}, \mathrm{n}}^{\mathrm{s}} \sin (2 \pi(\mathrm{m} u+\mathrm{n} v)),
$$

where $\mathrm{i}=\varphi, R, Z, s$ being the normalized toroidal flux, $u$ the poloidal angle and $v$ the toroidal angle. As an example, Fig. 6 shows the low-n part $(-10 \leq n \leq 10)$ of the Fourier spectrum of the asymmetric radial component $b_{R, \mathrm{~m}, \mathrm{n}}^{\mathrm{s}}$. Increasing the number of TBMs and distributing them equidistantly would not only eliminate these low-n harmonics in the ripple field, but also the overall magnitude of the field would be strongly reduced within the plasma. It is the low nharmonics with $n= \pm 1, \pm 2$ that are mainly responsible for the following observations.

As described in Sections 2 and 3, axisymmetric equilibria and 3D free-boundary equilibria which take the toroidal field ripple into account have been computed for $\langle\beta\rangle=2.24,3.66$ and 
4.67\%. For all these cases, the VMEC/NEMEC code yielded a fully converged solution. However, including the modifications of the ripple field by the TBMs the situation is different. A converged solution can only be found for $\langle\beta\rangle=2.24 \%$. For the two higher $\beta$-values the computations only converge in the beginning, but then a continuous increase of the $n=1$ Fourier harmonics sets in. No wall, free-boundary stability studies of the axisymmetric equilibria performed with the CASTROR_FLOW code [21] show that the high- $\beta$ equilibria are strongly unstable with respect to $\mathrm{n}=1$ ideal kink modes. The growth rates are $\gamma=1127301 / \mathrm{s}$ for $\langle\beta>=3.66 \%$ and $\gamma=1775801 / \mathrm{s}$ for $\langle\beta\rangle=4.67 \%$, while the equilibrium with $\langle\beta\rangle=2.24 \%$ is almost stable. Its growth rate is $\gamma=20831 / \mathrm{s}$. That is, a $3 \mathrm{D}$ free-boundary equilibrium reconstruction including low-n perturbation fields is not possible with the VMEC/NEMEC code for an unstable high- $\beta$ plasma.

The ferritic inserts decrease the toroidal field ripple considerably. Only in a small region at the low-field side close to the plasma boundary the effect of the ferritic inserts is small (see Figs 5 and 7c). However, the TBMs lead to a local increase of the ripple, as illustrated in Fig. 7. Fig. 7a shows the toroidal field ripple between $\varphi=20$ and $30^{\circ}$ (position of a TBM), while Fig. 7c presents the ripple between $\varphi=180$ and $190^{\circ}$, that is, far away from the TBMs. The ripple has been determined for the axisymmetric equilibrium field $(<\beta\rangle=2.24 \%)$ plus superimposed modified vacuum ripple field. Furthermore, in Fig $7 \mathrm{~b}$ and Fig. $7 \mathrm{~d}$ the ripple differences, $\Delta \delta$, between the 3D equilibrium fields and these fields are presented. Again the differences are negligibly small. It is interesting to note however that the plasma currents decrease the ripple in the neighbourhood of the TBMs but increase it everywhere else.

The low-n field components of the TBMs not only prohibit a stable equilibrium solution for higher $\beta$-values, they also lead to island formation. Figure 8 shows a Poincare plot of the axisymmetric equilibrium field $(\langle\beta\rangle=2.24 \%$ ) with superimposed field perturbations. Even close to the magnetic axis, small magnetic islands are formed at rational $q$-surfaces, and the boundary region is stochastizised because of overlapping islands. Typical island sizes are from inside outwards: $(3,1) \approx 2 \mathrm{~cm},(5,2) \approx 1 \mathrm{~cm},(3,1) \approx 1 \mathrm{~cm},(4,1) \approx 0.6 \mathrm{~cm}$ etc.. These island widths are of approximately the same size as the banana widths of the trapped thermal ions. In Fig. 9 two banana orbits of deuterons with $E=5 \mathrm{keV}$ and $E=2.5 \mathrm{keV}$ are plotted together with the magnetic islands. Since the island widths are comparable to the banana widths, the pressure profile will not be completely flat inside the islands. Taking into account that plasma rotation will shield the vacuum fields for higher $\beta$-values, the induced islands are not expected to serve as seed islands for neoclassical tearing modes (NTMs). However, the corresponding perturbation fields might cause locked magnetic islands during the current ramp up phase or reduce plasma rotation and cause locking of resistive wall modes in high- $\beta$ advanced scenario operation. 


\section{Summary and conclusions}

Non-axisymmetric equilibrium fields have been computed for ITER scenario 4 using (i) the standard tokamak method, that is, axisymmetric equilibrium fields plus superimposed 3D perturbation fields, and (ii) the 3D free-boundary VMEC/NEMEC code. Comparing the flux surface geometry and the magnetic field ripple, only negligibly small differences have been found, even for high $\beta$-values. The observed maximum ripple differences are smaller than $10 \%$. Therefore, we don't expect a significant effect concerning particle losses, if self-consistent 3D equilibrium fields are used for those studies. Furthermore, the VMEC/NEMEC code assumes nested flux surfaces, that is, islands generated by the TBM field are suppressed. With respect to this problem, the standard tokamak method seems to yield a more realistic magnetic field. For a completely self-consistent $3 \mathrm{D}$ equilibrium computation, which also takes island formation into account, the HINT [22] or PIES [23] codes would have to be used. The computation of 3D free-boundary equilibria with the VMEC/NEMEC code requires an enormous numerical effort, which would even be further increased by using the HINT or PIES code. However, since the observed plasma response to ripple and TBM perturbation fields is negligibly small, such a numerical effort does not seem to be appropriate for these kind of perturbations.

The convergence problems which occur for high-beta equilibrium computations including the TBM perturbation field indicate that the low-n components of this field have a destablizing effect. This is in agreement with previous findings that tokamaks are highly sensitive to nonaxisymmetric magnetic perturbations due to currents outside the plasma (see for e.g. [2, 24]). So, besides the effect of TBMs with respect to particle confinement $[7,12,13]$, also their consequences for the stability have to be investigated in detail.

In the boundary region, TBM magnetic fields have a similiar effect as the field of ELM control coils. Even if the planned ELM control coils cause a much stronger stochastizisation and larger islands [4], the effect of the TBMs should be included in those ELM studies. Finally, it should be noted that the TBM induced perturbations will influence the discharge from the very beginning.

\section{Acknowledgements}

We would like to thank S. Medvedev for providing the ITER equilibrium and coil data, and K. Shinohara for the magnetic field produced by TBMs and ferritic inserts. Furthermore, we would like to thank T. Kurki-Suonio and her team for many interesting discussions. 


\section{References}

[1] Buttery R J et al. 1999 Nucl. Fusion 391827.

[2] Scoville J T and La Haye R J 2003 Nuclear Fusion 43250.

[3] Evans T E et al. 2006 Nature Physics 2419.

[4] Bécoulet M et al. 2008 Nucl. Fusion 48024003 doi:10.1088/0029-5515/48/2/024003.

[5] Shinohara K et al. 2003 Nuclear Fusion 43586.

[6] Shinohara K et al. 2007 Nuclear Fusion 47997

doi:10.1088/0029-5515/47/8/034.

[7] Shinohara K et al. 2009 Fusion Engineering and Design 8424

doi:10.1016/j.fusengdes.2008.08.040.

[8] Villone F et al. 2008 35th EPS Conf. on Contr. Fusion and Plasma Phys. ECA 32D P2.080, Heraklion, Greece, http://epsppd.epfl.ch/Hersonissos/pdf/P2_080.pdf.

[9] Strumberger E, Merkel P, Sempf M and Günter S 2008 Phys. Plasmas 15056110 doi:10.1063/1.2884579.

[10] Goldstone R J and Towner H H 1981 J. Plasma Phys. 26283.

[11] Goldstone R J, White R B and Boozer A H 1981 Phys. Rev. Lett. 47647.

[12] Kurki-Suonio T et al. 2009 Nucl. Fusion 49095001 doi:10.1088/0029-5515/49/9/095001.

[13] Kramer G J, White R B, Nazikian R and Berk H L in Fusion Energy 2008 (Proc. 22nd Int. Conf. Geneva, 2008) (Vienna: IAEA) CD-ROM file IT/P6-3 and http://wwwnaweb.iaea.org/napc/physics/FEC/FEC2008/html/index.htm).

[14] Johnson J L and Reiman A H 1988 Nucl. Fusion 281116.

[15] Suzuki Y, Nakamura Y and Kondo K 2003 Nuclear Fusion 43406.

[16] Spong D A et al. in Fusion Energy 2008 (Proc. 22nd Int. Conf. Geneva, 2008) (Vienna: IAEA) CD-ROM file TH/3-4 and http://www-naweb.iaea.org/napc/physics/FEC/FEC2008/html/index.htm. 
[17] Strumberger E, Günter S, Schwarz E, Tichmann C and the ASDEX Upgrade Team 2008 New J. Phys. 10023017 doi:10.1088/1367-2630/10/2/023017.

[18] Hirshman S P and Lee D K 1986 Comput. Phys. Commun. 39161.

[19] Hirshman S P, van Rij W I, Merkel P 1986 Comput. Phys. Commun. 43143.

[20] Merkel P 1987 Nucl. Fusion 27867.

[21] Strumberger E, et al. 2005 Nucl. Fusion 451156.

[22] Harafuji K et al. 1989 Journal of Computational Physics 81167.

[23] Reiman A H and Greenside H S 1986 Computer Physics Communications 43157.

[24] Park J, Boozer A H and Glasser A H 2007 Phys. Plasmas 14052110. 


\section{FIGURE CAPTIONS}

Fig. 1: (a) flux surfaces, (b) pressure profiles, and (c) $q$-profile for various $\beta$-values, $<\beta>=2.24 \%$ (solid line), $\langle\beta>=3.66 \%$ (dashed line), and $\langle\beta>=4.67 \%$ (dashed-dotted line) of ITER scenario 4 equilibria.

Fig. 2: (a) toroidal field ripple, $\delta_{v}$, of the axisymmetric equilibrium field $(<\beta\rangle=2.24 \%$ ) with superimposed vacuum ripple field, and (b) corresponding ripple difference, $\Delta \delta$, between the $3 \mathrm{D}$ equilibrium field and this field. Furthermore, the ripple differences are shown for $(\mathrm{c})<\beta>=3.66 \%$, and $(\mathrm{d})<\beta>=4.67 \%$.

Fig. 3: Enlargement of outer midplane region of Fig. $2 d$ with superimposed normalized pressure profile (solid curve).

Fig. 4: (a) enlargement of the outer midplane region showing a flux surface of the 3D equilibrium at various toroidal angles ( $\varphi=0$ (left), 5 (middle/red), 10 (right) and $15^{\circ}$ (middle/blue)). (b) Poincaré plot of a field line at the same toroidal angles. This field line is started at co-ordinates on the flux surface and is traced in the corresponding axisymmetric equilibrium field with superimposed vacuum ripple field.

Fig. 5: Vacuum ripple field components (solid lines) and by TBMs and FIs modified components (dashed lines) as function of the toroidal angle for a representative co-ordinate point $(\mathrm{R}, \mathrm{Z})$.

Fig. 6: Low-n part of the Fourier spectrum of the asymmetric radial component, $b_{\mathrm{R}, \mathrm{m}, \mathrm{n}}^{\mathrm{s}}$, of the modified vacuum ripple field at the plasma boundary $(* \mathrm{~m}=0, \diamond \mathrm{m}=1, \triangle$ $\mathrm{m}=2, \circ \mathrm{m}=3, \nabla \mathrm{m}=4, \mathrm{X} \mathrm{m}=5,+\mathrm{m}=6)$.

Fig. 7: Toroidal field ripple, $\delta_{v}$, of the axisymmetric equilibrium field $(<\beta>=2.24 \%)$ with superimposed modified vacuum ripple field between (a) $\varphi=20$ and $30^{\circ}$, and (c) $\varphi=180$ and $190^{\circ}$. (b) and (d) show the corresponding ripple differences, $\Delta \delta$, between the $3 \mathrm{D}$ equilibrium fields and these fields.

Fig. 8: Poincaré plot of the axisymmetric equilibrium field $(<\beta\rangle=2.24 \%)$ with superimposed modified vacuum ripple field. (a) total view, and (b) enlargement of the lower X-point region.

Fig. 9: Enlargement of the low-field side region of Fig. 8a. Additionally to the magnetic islands, the figure presents the orbits of trapped thermal deuterons (solid lines) with $E=5 \mathrm{keV}$ and $E=2.5 \mathrm{keV}$. The numbers indicate the island type. 

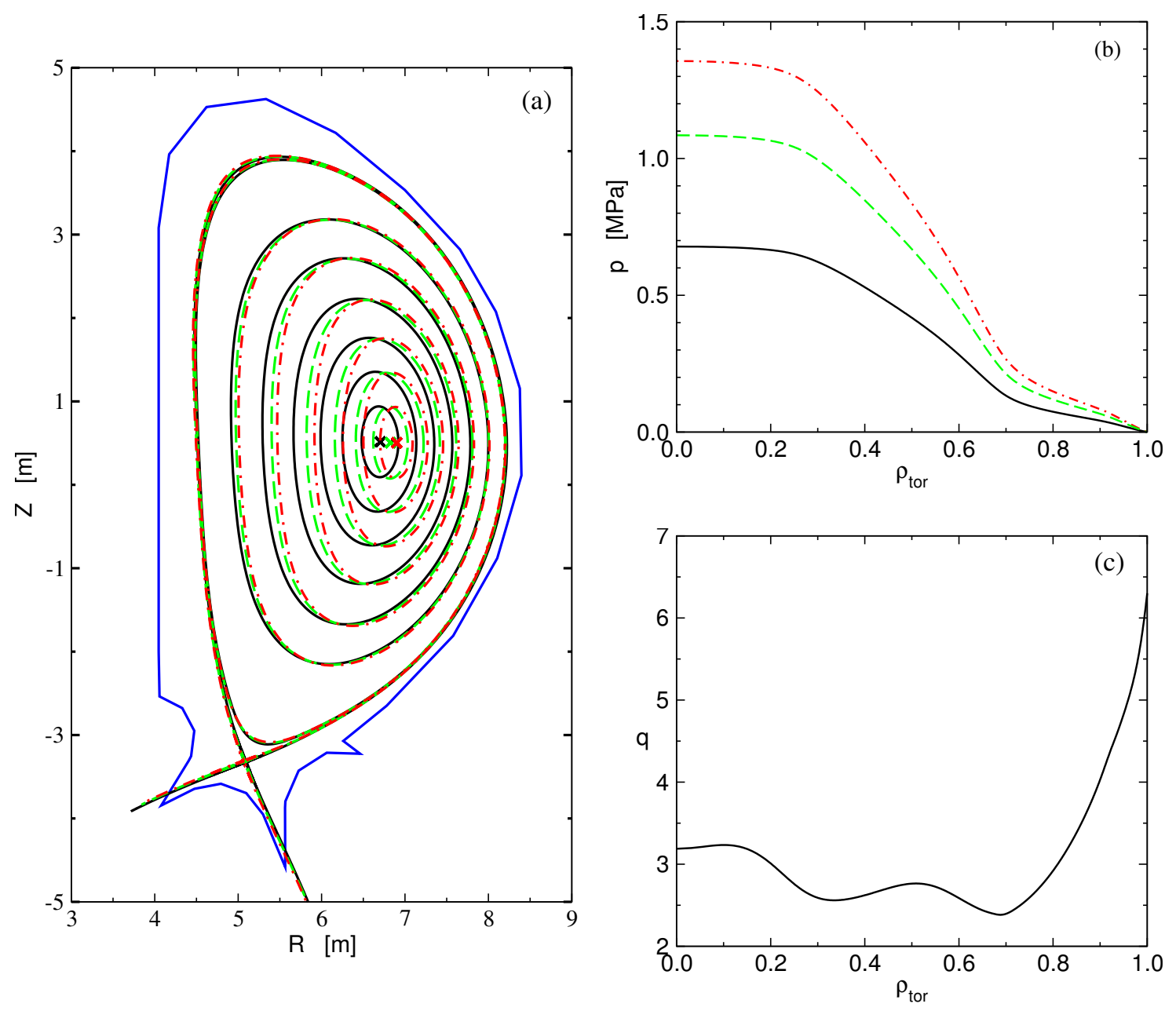

FIGURE 1 

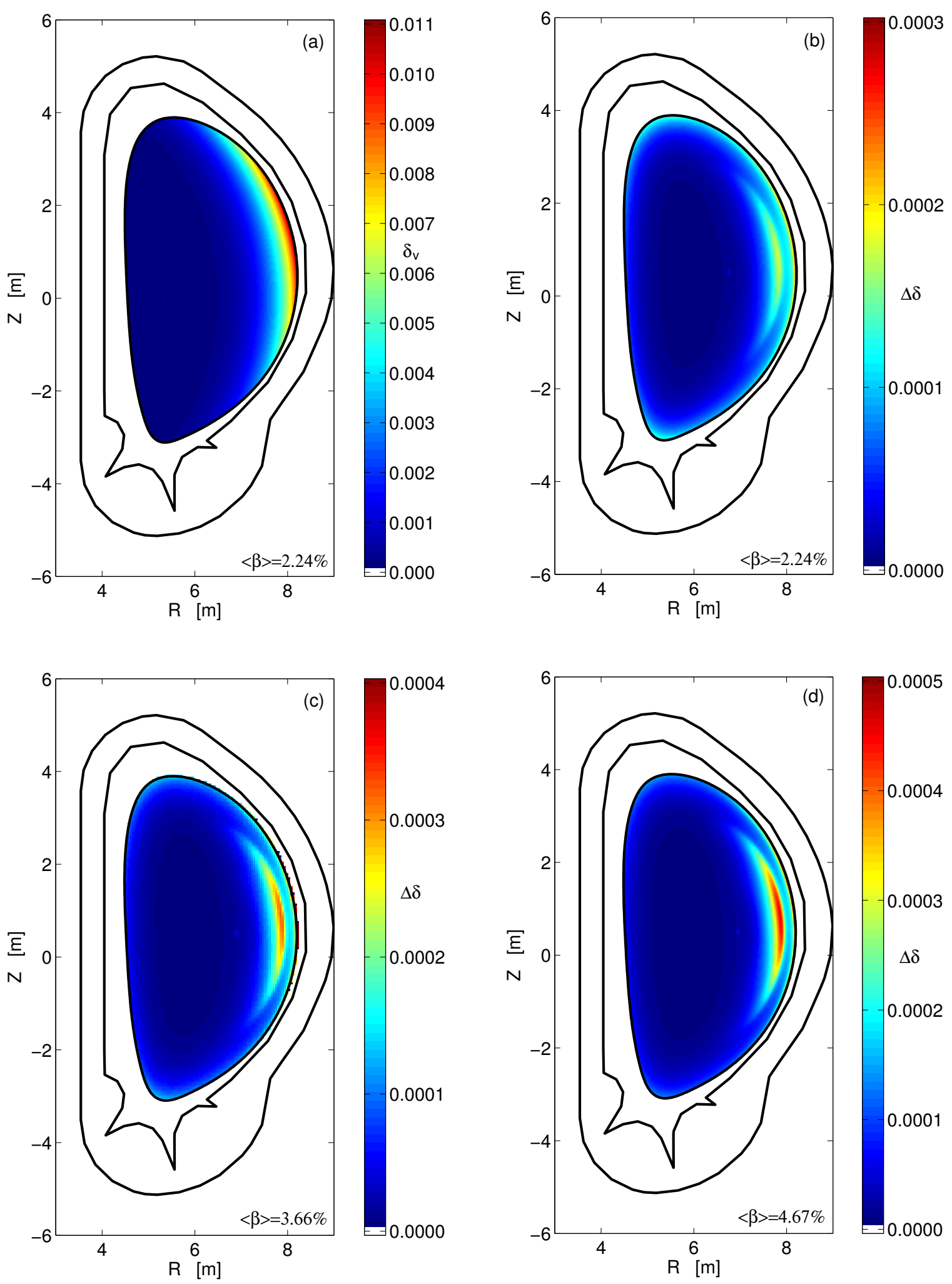

FIGURE 2 


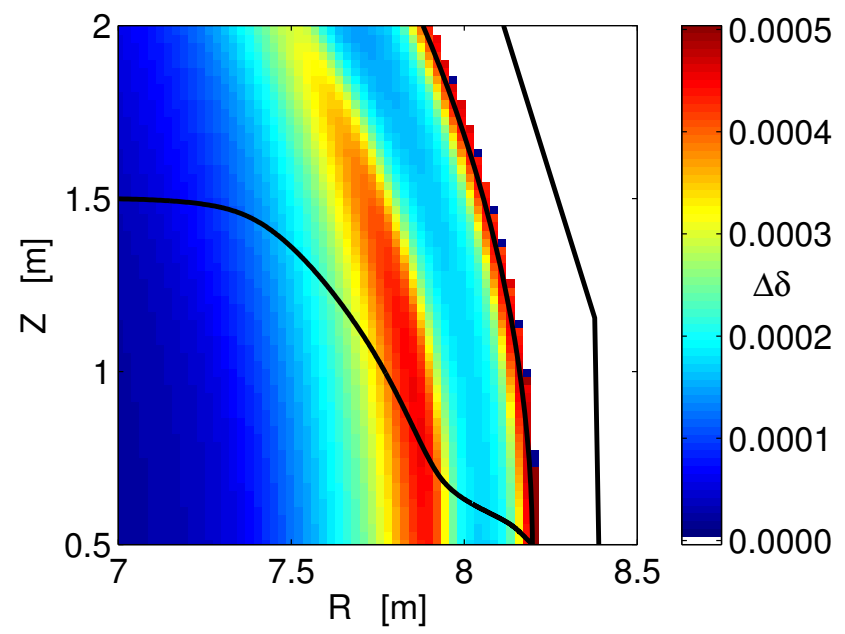

FIGURE 3 

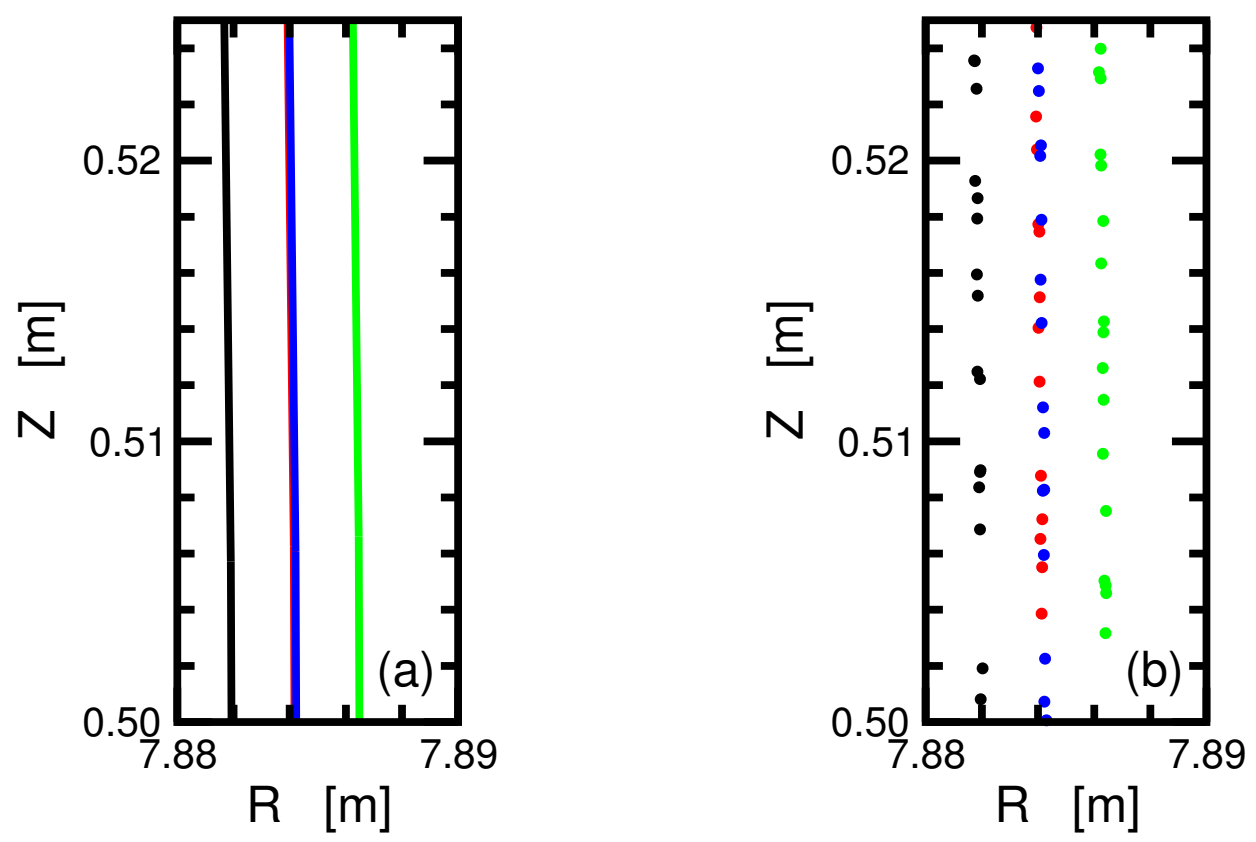

FIGURE 4 

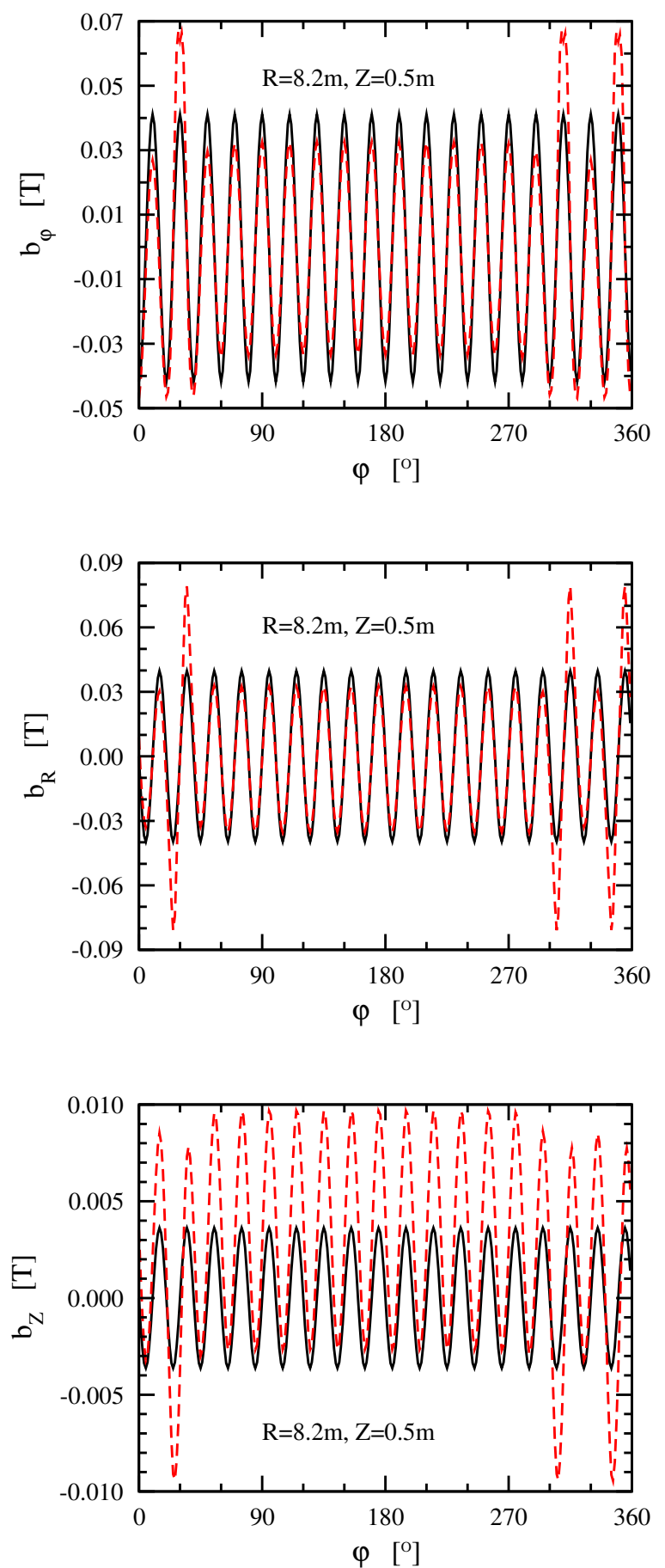

FIGURE 5 


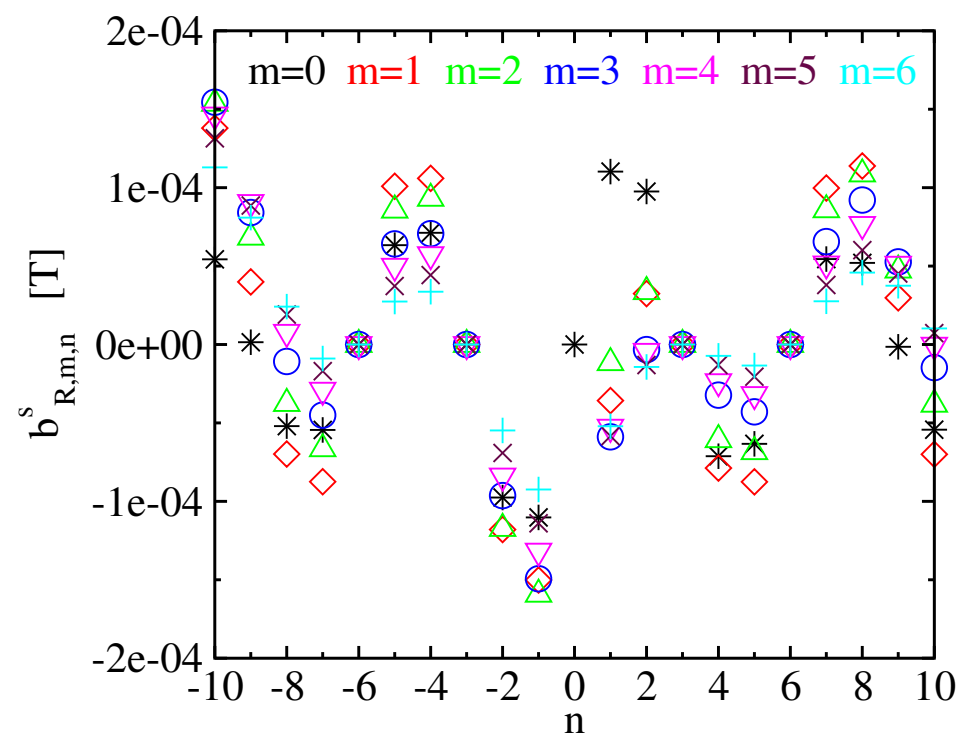

FIGURE 6 

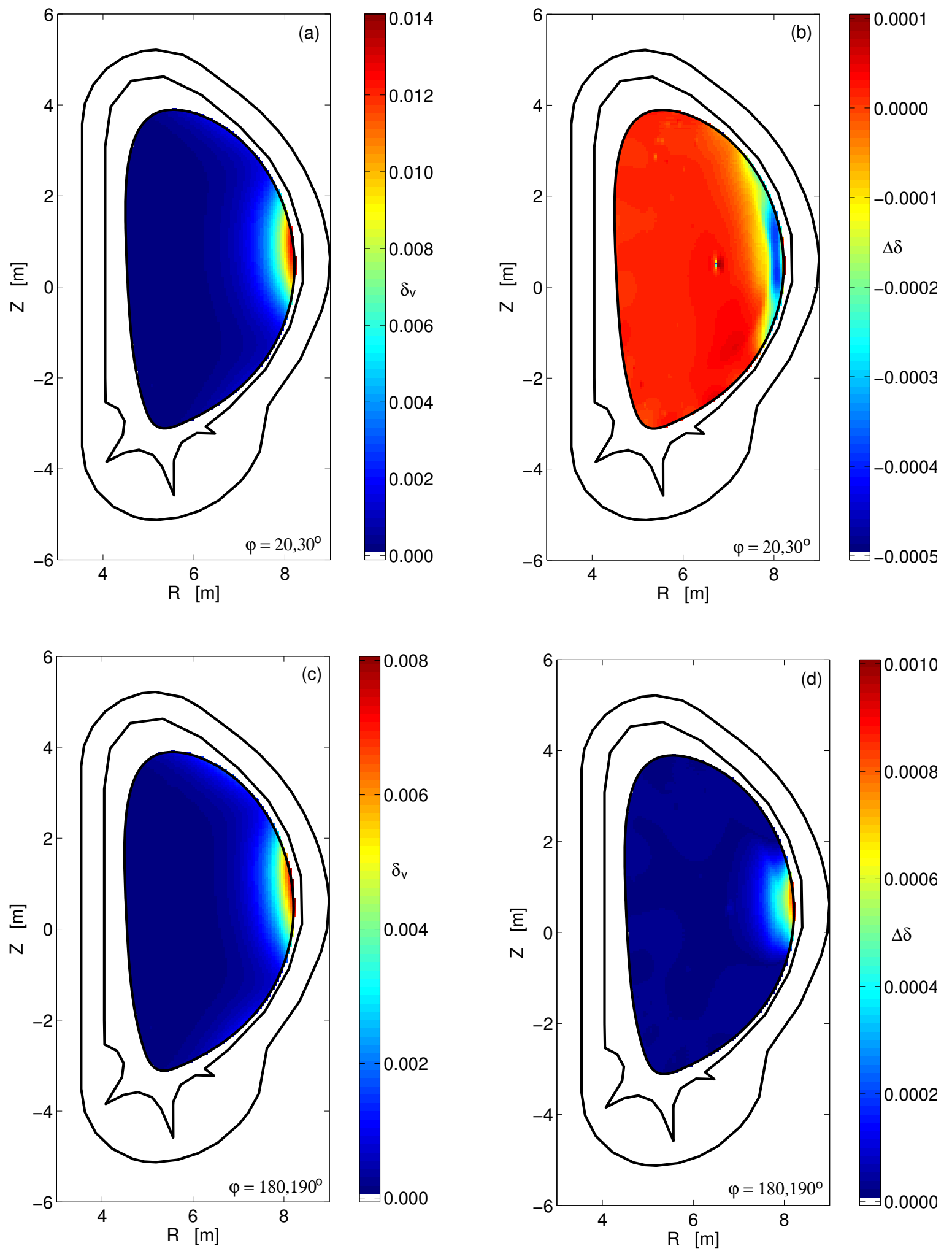

FIGURE 7 

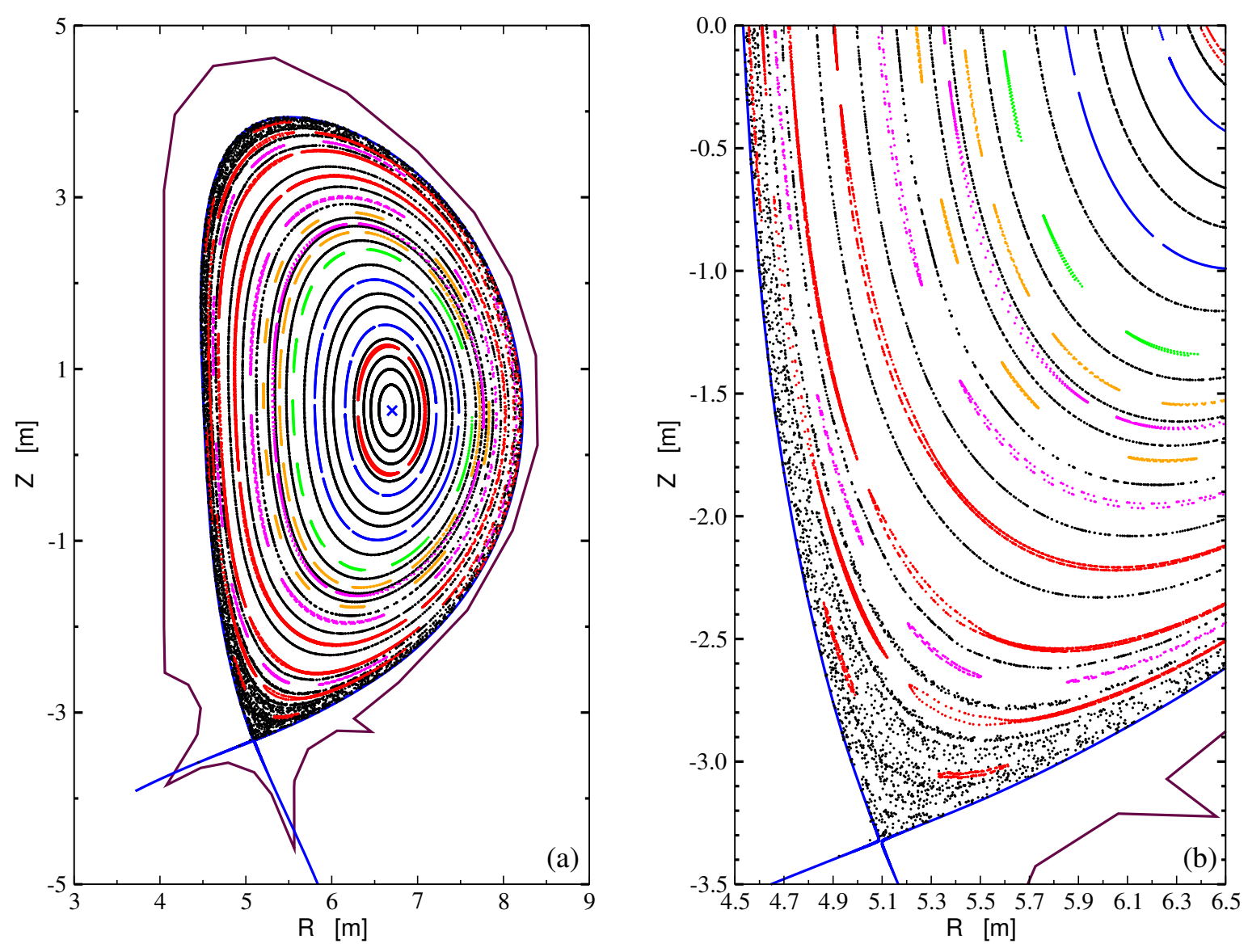

FIGURE 8 


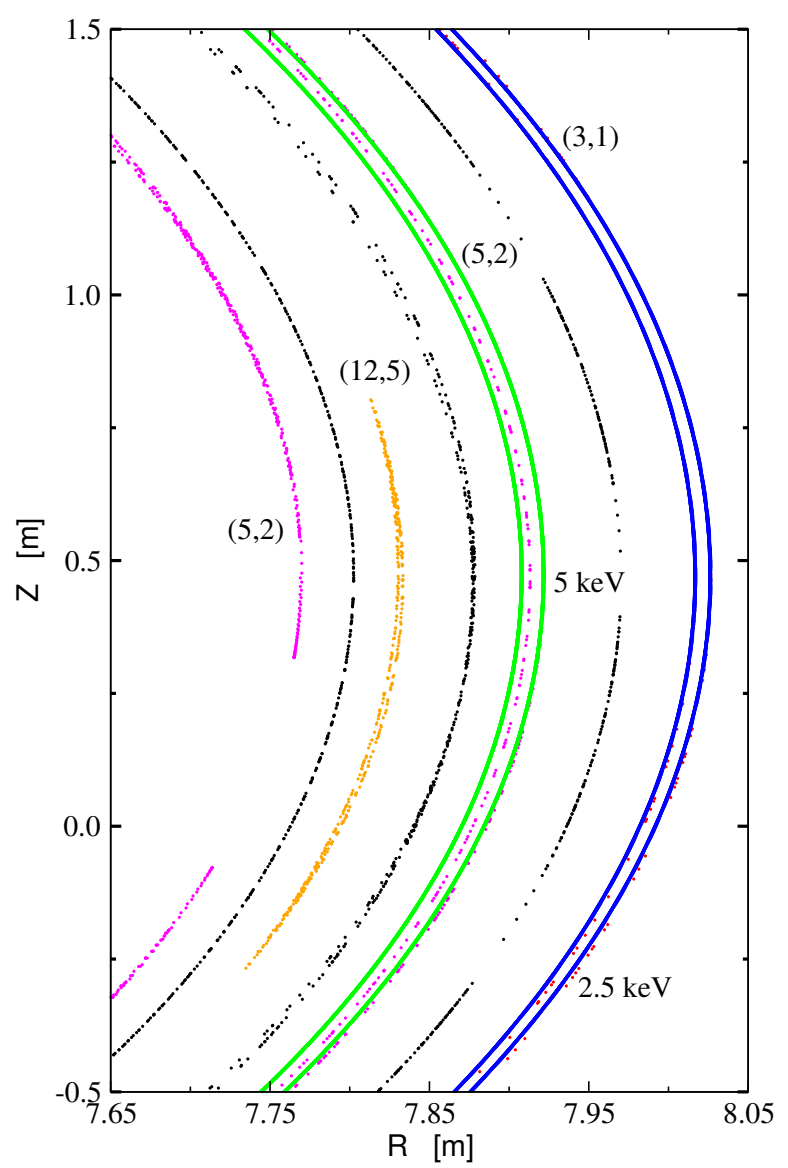

FIGURE 9 
Table 1. Currents in the poloidal field (PF) coils for equilibrium $\langle\beta\rangle=2.24 \%\left(J_{1}\right)$,

\begin{tabular}{lrrr}
\multicolumn{4}{l}{\langle\rangle$=3.66 \%\left(J_{2}\right)$ and $\langle\beta\rangle=4.67 \%\left(J_{3}\right)}$. \\
\hline \hline PF coils & $J_{1}(\mathrm{MA})$ & $J_{2}(\mathrm{MA})$ & $J_{2}(\mathrm{MA})$ \\
\hline PF1 & -3.299 & -3.299 & -3.299 \\
PF2 & 0.787 & 0.686 & 0.668 \\
PF3 & 4.422 & 5.201 & 5.667 \\
PF4 & 3.093 & 3.943 & 4.464 \\
PF5 & 5.246 & 5.052 & 4.972 \\
PF6 & -14.163 & -14.163 & -14.163 \\
CS3U & -6.548 & -6.548 & -6.548 \\
CS2U & -0.278 & 1.568 & 2.805 \\
CS1U & 9.840 & 7.461 & 6.173 \\
CS1L & 9.840 & 9.757 & 9.849 \\
CS2L & -3.615 & -2.001 & -0.962 \\
CS3L & -0.689 & -0.689 & -0.689 \\
\hline \hline
\end{tabular}

